WellBeing International

WBI Studies Repository

$1-1997$

\title{
Language-Naive Chimpanzees (Pan troglodytes) Judge Relations Between Relations in a Conceptual Matching-to-Sample Task
}

\author{
Roger K.R. Thompson \\ Franklin and Marshall College \\ David L. Oden \\ La Salle University \\ Sarah T. Boysen \\ The Ohio State University
}

Follow this and additional works at: https://www.wellbeingintlstudiesrepository.org/acwp_asie

Part of the Animal Studies Commons, Comparative Psychology Commons, and the Other Animal

Sciences Commons

\section{Recommended Citation}

Thompson, R. K., Oden, D. L., \& Boysen, S. T. (1997). Language-naive chimpanzees (Pan troglodytes) judge relations between relations in a conceptual matching-to-sample task. Journal of Experimental

Psychology: Animal Behavior Processes, 23(1), 31.

This material is brought to you for free and open access by WellBeing International. It has been accepted for inclusion by an authorized administrator of the WBI Studies Repository. For more information, please contact wbisr-info@wellbeingintl.org.

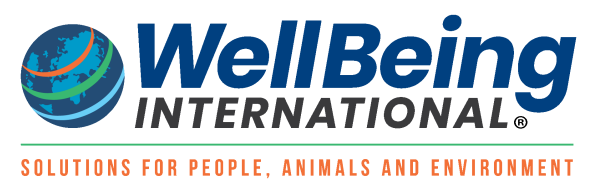




\title{
Language-Naive Chimpanzees (Pan troglodytes) Judge Relations Between Relations in a Conceptual Matching-to-Sample Task
}

\author{
Roger K.R. Thompson ${ }^{1}$, David L. Oden ${ }^{2}$, and Sarah T. Boysen ${ }^{3}$ \\ ${ }^{1}$ Franklin and Marshall College \\ ${ }^{2}$ La Salle University \\ ${ }^{3}$ Ohio State University
}

\begin{abstract}
Three chimpanzees with a history of conditional and numeric token training spontaneously matched relations between relations under conditions of nondifferential reinforcement. Heretofore, this conceptual ability was demonstrated only in language-trained chimpanzees. The performance levels of the languagenaive animals in this study, however, were equivalent to those of a 4th animal-Sarah-whose history included language training and analogical problem solving. There was no evidence that associative factors mediated successful performance in any of the animals. Prior claims of a profound disparity between language-trained and language-naive chimpanzees apparently can be attributed to prior experience with arbitrary tokens consistently associated with abstract relations and not language per se.
\end{abstract}

Early reports by some investigators indicated that language-trained chimpanzees differed profoundly in their problem-solving skills from chimpanzees who had not had such training (D. Premack, 1983a, 1983b, 1984, 1986; Savage-Rumbaugh, 1986). Language-trained chimpanzees solved conceptual problems that language-naive chimpanzees failed. D. Premack's language-trained animals, for example, reportedly were superior to the language-naïve animals on problems that required reasoning about relations between relations but that could not be solved merely on the basis of correspondence of physical features.

The discrepancy between language-trained and language-naïve chimpanzees reported by $\mathrm{D}$. Premack was based originally on the performances of seven African-born chimpanzees raised from infancy by human caregivers; three of the seven had received language training and four had not. Premack argued that the disparity in performance between these two groups of animals on such tasks could not be attributed to either age differences or their relative test sophistication (cf. D. Premack, 1984). Rather this profound disparity could be attributed only to whether or not an animal had received language training as originally described by D. Premack (1976). Short strings of rule-governed plastic words formed the physical basis of this language, which the chimpanzees used in both comprehension and production. See 
D. Premack and Premack (1994) for a summary of the procedure and D. Premack (1976) and A. J. Premack and Premack (1972) for extended examples.

One such task that differentiated between language-trained and language-naive chimpanzees in D. Premack's research program was a modified version of an analogy problem called conceptual matchingto-sample. Given AA as sample, for example, the correct response is to choose a BB rather than an EF alternative. Given CD as sample, the correct alternative is EF and not BB. Each letter within a pair refers here, and throughout this article, to a discrete object. Hence, an AA sample might consist of a pair of physically identical cups and BB to a pair of identical shoes. CD and EF might refer to pairs of nonidentical objects consisting, respectively, of a bottle top paired with a clothespin and a pen paired with a padlock.

Because the individual objects used to construct the sample and alternatives are physically different, successful matching cannot be based on the correspondence of physical features. Instead, successful performance of a conceptual matching problem must be based on the correspondence of two abstract relations. In this case, an animal is required to judge one pair of identical objects as instantiating the same within-pair relationship as another pair of identical objects. Likewise, the animal has to judge one pair of nonidentical objects as instantiating the same within-pair relationship as another pair of nonidentical objects (see Thompson \& Oden, 1996).

It is clear from D. Premack's early discussions (1983a, 1983b, 1984, 1986) that he considered prior practice with linguistic strings per se as necessary if a chimpanzee were to master conceptual relational matching and related tasks. In his later writings, D. Premack $(1988,1992)$ indicated that, although some aspects of language training with discursive sequences of symbols were sufficient, none was a necessary prerequisite for at least some minimal competence level in judging relations. For example, D. Premack asserted that a procedure he labeled "dogged training" alone could enable a language-naive chimpanzee to match like proportions of different objects (e.g., $1 / 4$ apple $=1 / 4$ glass of water; see D. Premack, 1988, p. 54). We assume that "dogged training" in this setting meant persistent training involving very many trials, perhaps thousands, on a single problem using differential reinforcement; if so, then the description is apt. Oden, Thompson, and Premack (1990), using the same four animals identified by D. Premack (1988), found no evidence of conceptual-relational matching after extensive training of more than 1,000 trials per animal.

Premack's conclusion regarding the efficacy of "dogged training," however, is seemingly supported by results reported in an earlier study by Smith, King, Witt, and Rickel (1975). Those investigators presented data suggesting that one of two language-naive chimpanzees matched conceptual relations that the authors labeled as homogeneity (i.e., identity) and heterogeneity (i.e., nonidentity). However, the experimental design and presentation of results preclude a definitive conclusion as to the actual processes underlying the performance of this animal over the course of 5,000 trials (Thompson \& Oden, 1996).

Let us grant that Smith et al. (1975) and D. Premack (1988) found evidence of conceptual relational matching in language-naive chimpanzees after thousands of differentially reinforced trials. The contrast between the effectiveness of their procedures and the virtually immediate success of language-trained chimpanzees under conditions of nondifferential reinforcement serves to underscore the profound contribution of some aspect of language training.

Two fundamental questions arise regarding the causal importance of D. Premack's language training regimen for judgments of relational equivalence as described previously. First, in what way does 
language training change a chimpanzee? Second, what aspects of the training are responsible for the change?

We have reported evidence from infant chimpanzees pertinent to the first question (Oden et al., 1990). Specifically, infant chimpanzees who matched objects on the basis of physical appearance nevertheless could not match relations conceptually despite extensive training. However, the same four chimpanzees demonstrated, via differential handling in a familiarization-novelty task, that they perceived similarities and differences between exemplars of identity and nonidentity relationships despite their inability to judge the equivalence of such relations in a conceptual matching task. Thus, these findings imply that comprehension and production of functional symbolic sequences do not instill perceptual sensitivity to abstract relations. This sensitivity exists before any such training. Language training, then, apparently permits chimpanzees to express judgmentally what they otherwise only perceive.

Thompson and Oden (1993), discussing this disparity between perceptual and judgmental capacities, suggested that conceptual matching might emerge in a chimpanzee if the animal were provided with any concrete token that served as a representational tag for the relation. That is, provision of a token for "same" enables an animal to match pairs of objects despite the absence of any physical resemblance between them. According to this argument, the token or tag need not be imbedded within the context of a larger linguistic string. It need only be a functional icon onto which an abstract relation could be mapped. The implication then is that a string of elements is not necessary to represent certain prepositional conditions such as the abstract relations of sameness and difference.

D. Premack independently came to a similar position on the basis of his experience with a group of four captive-born chimpanzees studied from infancy to near puberty (D. Premack, 1988, 1992). In this latter work, he used a language-training regimen consisting of: (a) lexicon and (b) "sentence" acquisition. Introduction of plastic tokens representing the words same and different was the third step, which D. Premack (1988) concluded was the critical step in language training. Furthermore, he argued that the words same and different need refer during training to only physical similarity (e.g., "apple same apple"). This experience alone, D. Premack concluded, was sufficient for the chimpanzee to grasp that the distinction was broadly construed, applying equally well to abstract relations as it did physical resemblance. Nevertheless, he continued to speculate that the first two steps-lexicon and sentence training-may be "indirect prerequisites" for enabling an animal to express relational knowledge (D. Premack, 1988, p. 53). In this regard, his more recent stance was consistent with his earlier statements that the critical aspect of language training is extensive experience with "a linguistic string and the condition it describes" (D. Premack, 1984, p. 204) or in computing "the equivalence between a condition and a discursive representation of the condition" (D. Premack, 1984, p. 204).

The present study is relevant to this issue of whether such experience is a necessary prerequisite for conceptual-relational matching. Three of the animals, whose data are reported here, had not received the previously described language training nor had they been tested on a conceptual-relational matching task. These three chimpanzees, however, did have a history of conditional discrimination training using tokens and multiple pairs of objects. That is, a single pair of physically identical items (e.g., AA) was a discriminative stimulus for choosing an arbitrary heart-shaped token. Conversely, they had been rewarded for choosing an arbitrary diagonally shaped token after presentations of single nonidentity discriminative stimulus pairs (e.g., CD). The reflexive equivalence relation had not been trained or tested (i.e., "If heart, then choose AA"). In addition, these animals also had a history of counting in which they had been trained to associate Arabic numerals with corresponding numeric arrays (Boysen, 1993; Boysen \& Bernston, 1989). 
As noted, Thompson and Oden (1993) proposed that prior provision of a concrete tag for an abstract relation is sufficient for immediate conceptual-relational matching to occur in the absence of any explicit training on that task. If so, then chimpanzees with a history of conditional discrimination training, as described previously, should match conceptually on their first encounter with the problem. On the other hand, if explicit relational judgments additionally require exposure to structured strings of tokens or dogged training on a conceptual-relational matching task, then these same three animals should fail on their initial encounter with that problem.

\section{Method}

\section{General Design}

The present study consisted of three matching-to-sample problem sets. Each set consisted of a block of physical matching trials and a block of conceptual-relational matching trials. Identity and nonidentity pairs were used as samples and alternatives in both the physical and conceptual matching problems. In Table 1 , as elsewhere in this article, individual letters are used for expository purposes and refer to individual objects or digitized images that served as stimuli. In physical matching problems, both the identity and nonidentity samples consisted of pairs that were physically identical to the correct alternatives. For example, both MM and NO appeared as samples and as alternatives on all physical matching trials in Problem Set 1 . In conceptual-relational matching problems, both the identity and nonidentity samples consisted of pairs that differed physically from the alternatives. For example, in Problem Set 1, AA and EF appeared as samples and BB and CD always appeared as the corresponding matching alternatives.

The first problem set was intended to determine whether the animals could match conceptually despite their never having been trained on that particular relational problem. They were tested on conceptualrelational matching trials using nondifferential reinforcement in which all choices, both correct and incorrect, were rewarded. Nondifferential reinforcement was used to determine whether conceptual matching of relations between relations would occur spontaneously and to mitigate against rapid acquisition of a learning set, which might occur when differential reward is used (cf. Nissen, Blum, \& Blum, 1948; Oden, Thompson, \& Premack, 1988). The nondifferential reward procedure was used rather than an extinction procedure because the latter disrupts performance by chimpanzees. Extrinsic rewards, however, motivate a chimpanzee to continue a task independently of performance level (cf. Oden et al., 1988; Oden \& Thompson, 1992). The physical matching trial blocks were intended to provide a baseline for matching abilities under optimum conditions of physical identity and differential reinforcement. Hence, in contrast to conceptual-relational matching trials, correct physical matching responses were rewarded and incorrect responses were not.

The second problem set involving novel stimuli was intended to ensure that any conceptual matching performance demonstrated on the first problem set was not simply an artifact of the stimulus items used in the first problem set. Again, differentially reinforced physical matching trials served as a baseline. The third problem set was also intended as an additional test for conceptual-relational matching and further served to evaluate the possible role of associative processes hi performance of this task. Novel pairs were constructed using individual objects from the first two problem sets.

Recombining individual objects into novel alternatives for Problem Set 3 was intended to reveal whether earlier performances in the conceptual matching trials were mediated by associative processes rather than relational equivalence judgments. Decrements in performance would be expected in Problem Set 3 if the chimpanzees based their choices on simpler associative strategies rather than conceptual matching of relations between relations. On the other hand, no such decrement was predicted if performances were conceptually mediated. 
Table 1. Stimulus Pairs Used in Problem Sets 1 to 3

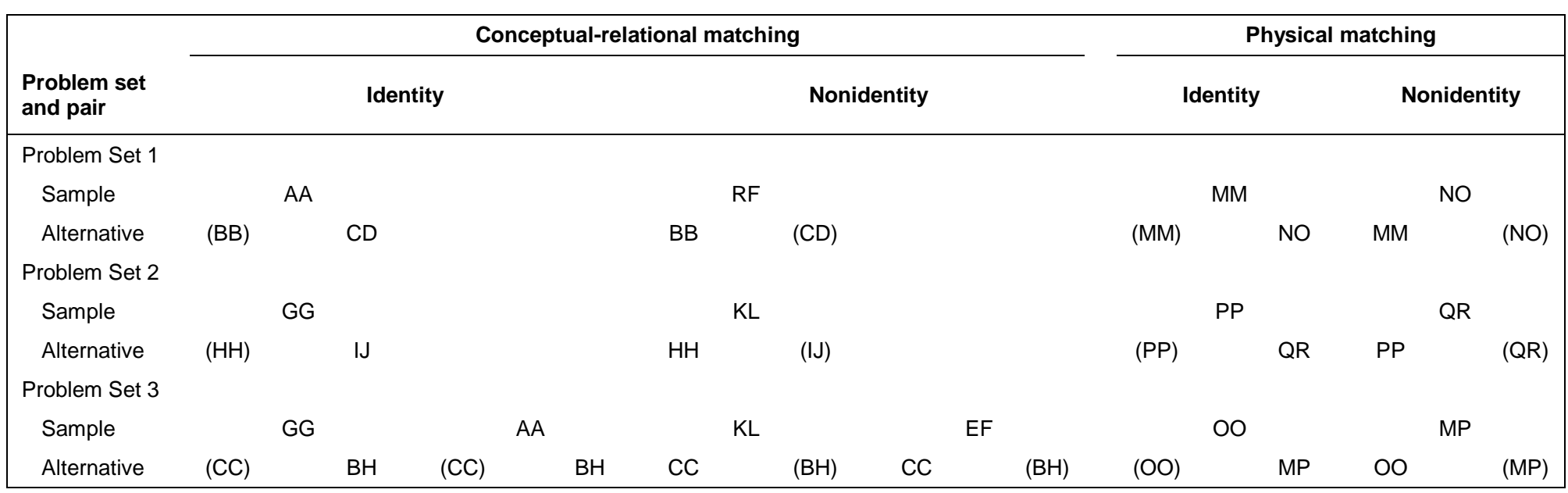

Note. Pairs in parentheses indicate correct alternative choices. Individual letters within a pair refer to discrete objects (e.g., A = yellow talcum powder can and $B=$ green tin cup).

\section{Animals}

One juvenile and 4 adult chimpanzees (Pan troglodytes) were included in this study. One adult, Sarah, had been trained previously on the symbolic language system developed by D. Premack (e.g., A. J. Premack \& Premack, 1972; D. Premack, 1976). She was one of the animals whose performance was commonly cited as evidence for the disparity between language-trained and language-naive chimpanzees. The 3 remaining adult chimpanzees -1 female (Sheba) and 2 males (Darrell and Kermit) included in the present study had a history of conditional discrimination training using tokens and multiple object pairs as described previously (Boysen, 1993; Boysen \& Bemston, 1989). A fifth juvenile male animal, Bobby, had received neither language training nor the conditional discrimination task.

All of the animals lived in a group housing complex at the Comparative Cognition Project facility at Ohio State University (e.g., see Boysen, 1992). They had access to both indoor and outdoor areas and had been taught to "take turns" at entering an adjacent test room for experimental sessions. They were neither food nor water deprived and could leave the testing session at any time by simply moving outside.

\section{Apparatus and Stimuli}

Because we were interested in immediate spontaneous matching, we deemed it best to use a procedure of stimulus presentation that was familiar to the animals. Hence, object pairs serving as samples were displayed on a narrow shelf on the experimenter's side of a Lexan window, which extended along one wall of the testing room. Rewards (preferred candies) were dispensed via a plastic tube projecting through the window. Digitized (i.e., PICT files) video images of the alternative choice pairs were presented to the animal on a color computer monitor (NEC, Japan) in the test room at right angles to the window. An IBM (Poughkeepsie, NY) microcomputer controlled presentation of the digitized choice stimuli and recorded the animal's choices as detected by a Carroll (Round Rock, TX) touch-frame attached to the monitor. Auditory feedback was also controlled by the computer. The procedure of using actual objects as samples and digitized images as choice alternatives further provided a potential assay of object-picture matching should the animals fail the conceptual matching task. 
Stimulus pairs for both physical and conceptual-relational matching problems were constructed from individual junk objects that differed in color, size, and shape. Sample pairs consisted of the objects themselves mounted on a cardboard base (e.g., a yellow talcum powder can mounted side by side with a physically identical talc can). Choice alternative pairs consisted of $76 \times 76-\mathrm{mm}$ digitized images of the junk object pairs. These were presented on the monitor, separated, edge to edge, by $76 \mathrm{~mm}$.

Six individual objects were used to construct the pairs presented in the initial conceptual problem set (see Table 1). An additional three objects made up the pairs used in the physical matching trials. Nine novel objects were used to construct the physical and conceptual samples and alternative pairs in Problem Set 2. The samples and alternatives for the physical matching trials in Problem Set 3 were constructed from three familiar objects arranged in novel combinations (see Table 1). The samples in the conceptual matching trials of Problem Set 3 were the same four pairs used in Problem Sets 1 and 2. The alternative stimulus pairs in the conceptual matching trials consisted of novel pairings of objects used in alternative pairs in Problem Sets 1 and 2 (see Table 1).

The individual objects were recombined in such a way as to reveal whether performances in the first two conceptual matching problem sets were mediated by associative rather than conceptual-relational links between samples and alternatives. First, objects in the novel identity alternatives of Problem Set 3 were drawn from the nonidentity pairs used in Problem Sets 1 and 2. Conversely, objects in novel nonidentity alternative pairs were drawn from previously used identity pairs. These rules were used for both conceptual and physical matching trials in the third problem set. The rules for constructing conceptual matching alternative pairs were further constrained as follows: For any particular sample in Problem Set 3 , at least one member of the correct alternative had previously appeared within the incorrect alternative associated with that specific sample in either Problem Set 1 or 2 . These rules permitted us to probe for the possible control of performances by stimulus specific associations.

For example, in Problem Set 1, Object A from sample pair AA might have become associated with Object $B$ of the correct BB alternative. Hence, in Problem Set 3, when AA was the sample, then B was one of the objects in the incorrect $\mathrm{BH}$ alternative. Likewise, in Problem Set 1, either the $\mathrm{C}$ or the $\mathrm{D}$ object within the nonidentity alternative might have become associated with either $\mathrm{E}$ or $\mathrm{F}$ of the corresponding nonidentity sample. Hence, in Problem Set 3, if EF was the sample, then Object C was used in the incorrect CC identity alternative. It is apparent then that any stimulus-specific associations acquired in Problem Sets 1 or 2 would encourage the chimpanzees to choose an incorrect alternative in Problem Set 3, resulting in performance decrements.

These stimulus construction rules also allowed us to probe for conditional associations between sample relations (i.e., identity or nonidentity) and a specific item within the correct alternative pairs. For example, in Problem Set 1 a chimpanzee may have chosen correctly not by matching relations with relations but by using an associative strategy of "If identity, choose B" and "If nonidentity, choose C." Hence, Objects B and $\mathrm{H}$, previously associated with identity samples AA and GG, respectively, were used in Problem Set 3 as the nonidentity alternative pair $\mathrm{BH}$. Likewise, Object $\mathrm{C}$, previously associated with the nonidentity sample EF, was used to construct the CC identity alternative in Problem Set 3. If prior performances had been controlled by conditional associations, then performance decrements would be expected in this third problem set.

\section{General Procedure}

Each test session consisted of a block of 16 physical matching trials and a block of 16 conceptualrelational matching trials. Blocks of physical and conceptual matching trials were counterbalanced between successive sessions in an ABBA design. Presentation order of the eight identity and eight 
nonidentity trials was randomized within each block. The criterion for successful performance on each problem set was $75 \%$ correct choices on both physical and conceptual matching trials on each of two consecutive sessions. Once an animal met this criterion, it advanced to the next problem set.

In both physical and conceptual matching-to-sample, a trial began with the experimenter displaying the sample pair to the subject through the observation window. The sample remained visible for the duration of the trial. After the animal had looked at the sample, both alternative pairs were displayed simultaneously on the monitor side by side. Spatial position of the alternatives was balanced over each 16-trial block. The animal indicated his or her choice by touching one of the alternative images on the screen, and the computer automatically recorded the animal's choice.

Computer-generated feedback for correct responses on physical matching trials consisted of a highpitched tone $(800 \mathrm{~Hz})$ followed by a candy reward and an approximately 20-s intertrial interval (in). Incorrect responses were followed by a low-pitched buzzer $(300 \mathrm{~Hz})$ and then the ITI. Nondifferential feedback was used on all conceptual matching-to-sample trials. That is, both correct and incorrect responses were accompanied by a middle-pitched beep $(500 \mathrm{~Hz})$ followed by a candy reward and then the ITI.

Tests for inadvertent cueing of the correct stimulus pair image by the experimenter were run after the completion of Problem Set 3. These included 16 "Clever Hans" conceptual matching trials during which a second experimenter placed the sample pair on the shelf outside the test window and then immediately stepped out of the testing room before the alternative choice pair images were presented on the touchscreen monitor. Experimenter 1 was seated behind a barrier out of sight of the animal and thus could not see the samples or the animals' responses. Once the animals had made a response via the computer touch-frame system, auditory feedback indicated that they had completed each trial, so that reinforcement could then be provided. If earlier performances had been a function of inadvertent cueing by the experimenter, then a decrement in performance might be expected. As indicated in the results reported next, this did not occur. Interestingly, in a previous study, language-naive infant chimpanzees failed to match relations between relations even when the experimenter explicitly pointed to the correct alternative (Oden et al., 1990).

\section{Results}

Two animals, Darrell and Sheba, reached criterion on the original problem set in two sessions, each consisting of one block of 16 physical matching trials and one block of 16 conceptual-relational matching trials. Sarah reached criterion by the third session, and Kermit reached criterion after the fourth session of 16 conceptual-relational and 16 physical matching trials. The fifth animal, Bobby, failed to meet criterion within four sessions. To confirm that criterion level performances were stable, an additional postcriterion session was run for the 3 animals that met criterion in fewer than four sessions. Performance levels did indeed remain stable, and results for the last two sessions completed by each animal are shown in Figure 1. Overall mean performance across the 4 animals that met criterion was $83.6 \%$ for physical matching and $84.4 \%$ for conceptual-relational matching.

We cannot explain why 2 animals performed above chance immediately under nondifferential reinforcement and the remaining subjects did not. The important point, however, is that they did reach criterion levels of conceptual-relational matching performance in as few as 32 trials and in no more than 64 nondifferentially reinforced trials. This finding stands in sharp contrast to the earlier described reports in which language-naive chimpanzees acquired conceptual-relational matching, if at all, only after thousands of differentially reinforced trials (cf., Oden et al., 1990; D. Premack, 1988; Smith et al., 1975). 
Figure 1. Percent correct physical (i.e., object) and conceptual (i.e., relations between relations) matching-tosample (MTS) judgments by chimpanzees on Problem Set 1. Judgments on physical MTS trials were differentially rewarded, whereas judgments on conceptual MTS trials were not.

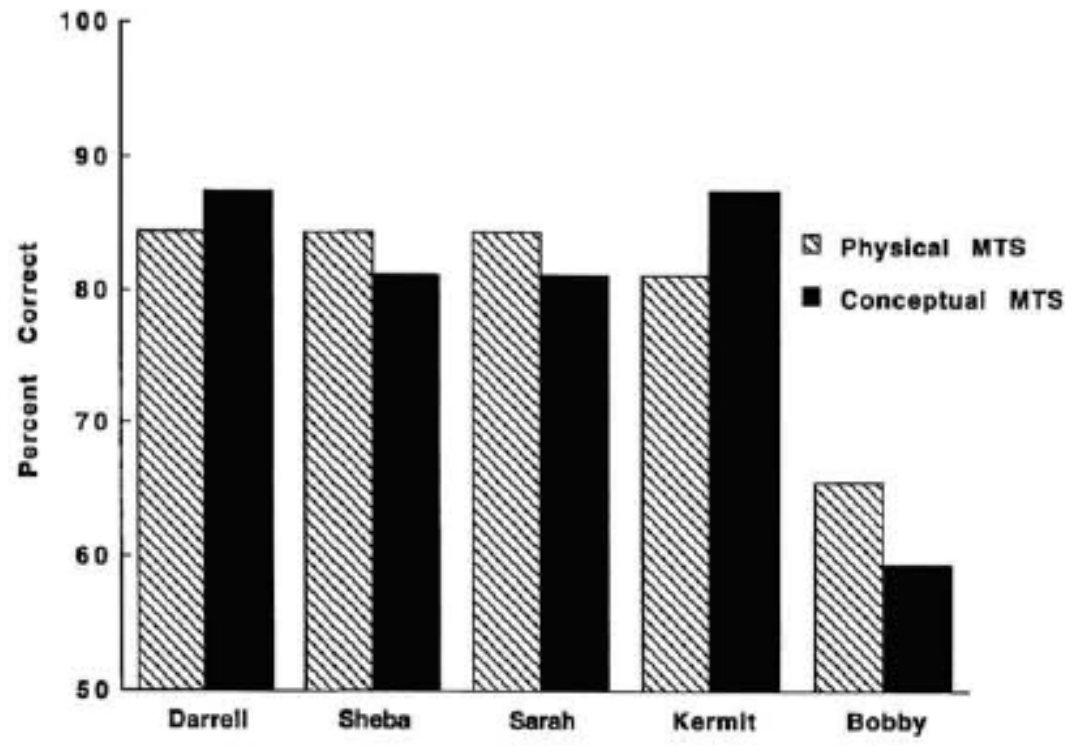

Figure 2. Percent correct physical (i.e., object) and conceptual (i.e., relations between relations) matching-tosample (MTS) judgments by chimpanzees on Problem Set 2. Judgments on physical MTS trials were differentially rewarded, whereas judgments on conceptual MTS trials were not.

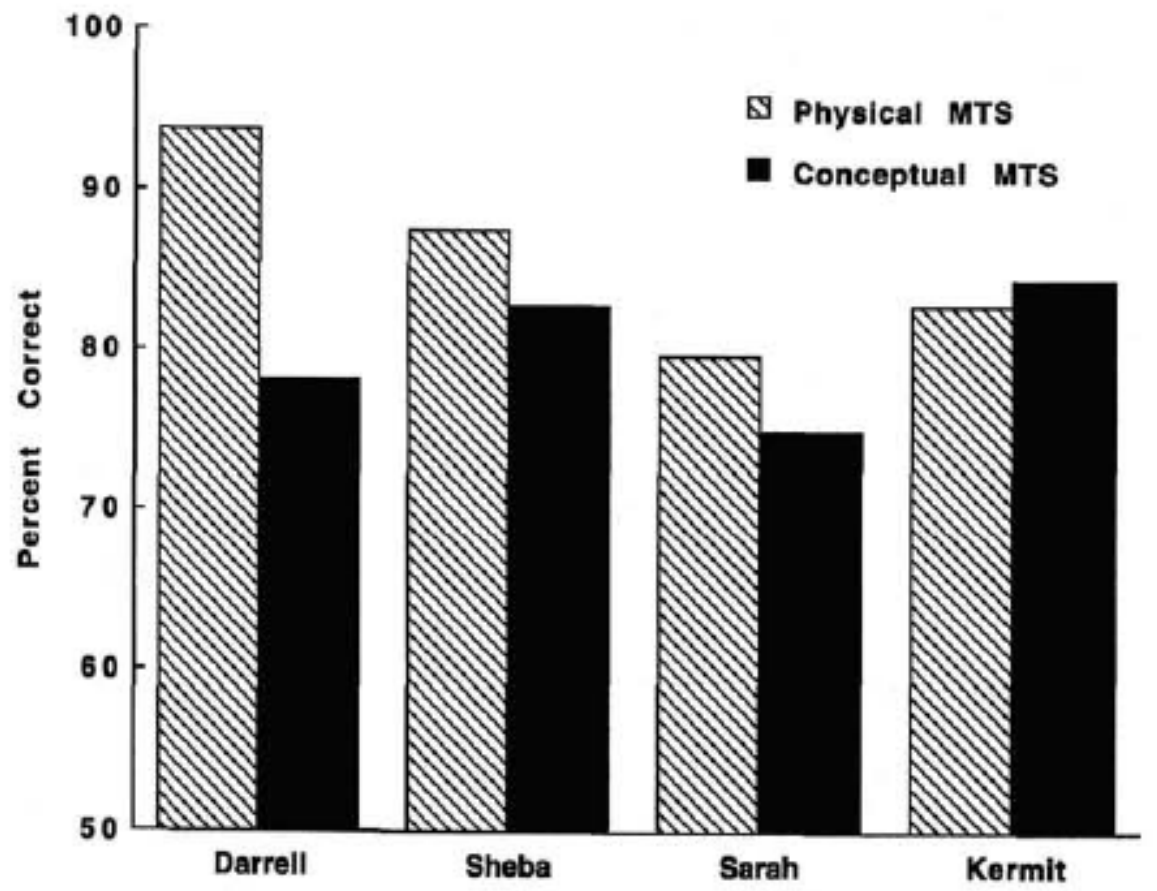


Figure 3. Percent correct physical (i.e., object) and conceptual (i.e., relations between relations) matching-tosample (MTS) judgments by chimpanzees on Problem Set 3 . Judgments on physical MTS trials were differentially rewarded, whereas judgments on conceptual MTS trials were not.

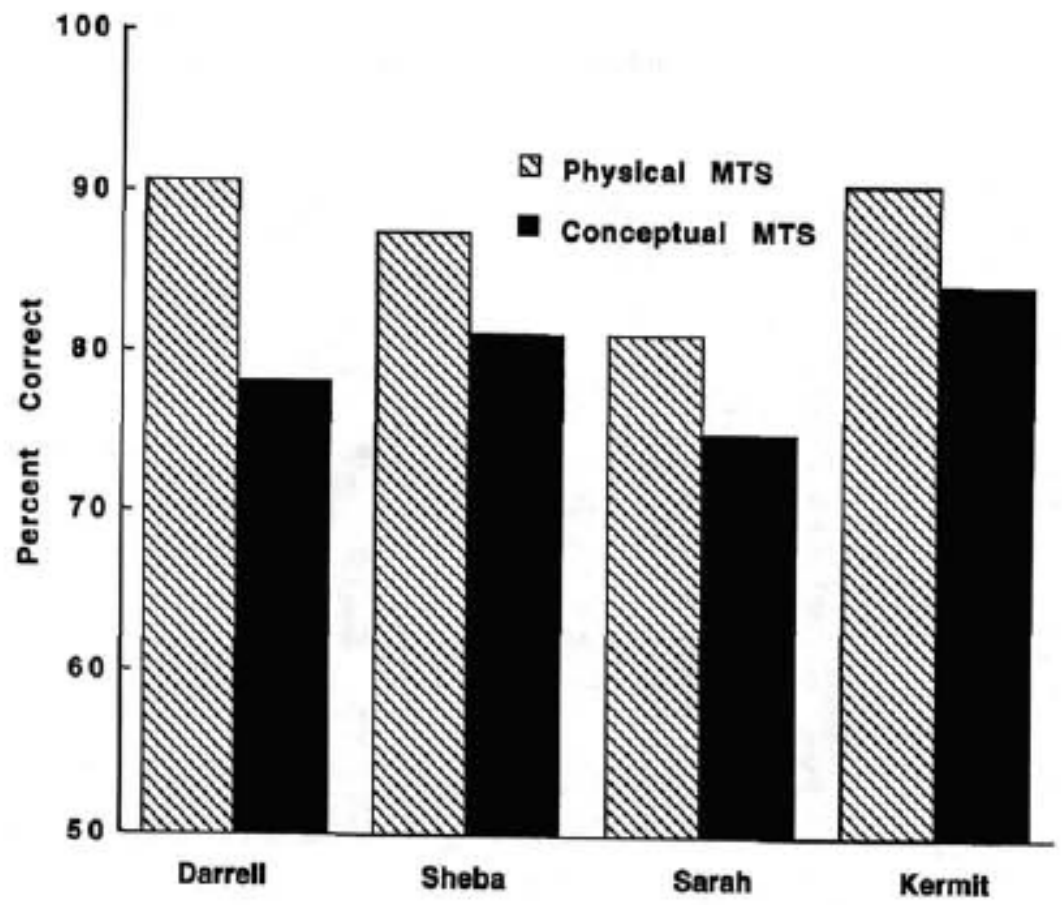

These data demonstrate that 4 of the 5 chimpanzees could physically and conceptually match pairs of objects with the correct alternative pair presented as images on a monitor screen. The conceptual matching of relations between relations occurred even in the absence of explicit instruction via differential reward. In conceptual-relational matching trials, the correct alternative was an image of a pair of objects that differed physically from the sample object pair but that instantiated the same relation (identity or nonidentity) as the pair of objects making up the sample. In the case of physical matching trials, the correct alternative pair was an image of the actual objects presented as the sample pair.

Both physical and conceptual matching performances continued without significant change in two novel problem sets under the same conditions of differential reward in the physical matching condition and nondifferential reward in the conceptual-relational matching condition. These data are shown in Figures 2 and 3. Overall mean physical matching performances across animals were $84.4 \%$ and $87.5 \%$ in Problem Sets 2 and 3, respectively. The corresponding overall mean performances for conceptual matching in Problem Sets 2 and 3 were $80.5 \%$ and $79.7 \%$, respectively. Overall mean correct performances on these two additional problems combined was $85.9 \%$ for physical matching and $80.1 \%$ for conceptual matching. The overall mean correct performance on ihe Clever Hans conceptual matching trials conducted in Problem Set 3 was $87.5 \%$.

The data strongly suggest that these four chimpanzees spontaneously judged the conceptual equivalence of relations between relations. A preliminary repeated measures analysis of variance (ANOVA; $2 \times 3 \times 2$ $\times 2$ ) with main factors of matching type (physical vs. conceptual), problem set (1,2, and 3), session (1 and 2), and within-session trial block (1 and 2) revealed no differences between overall performances in 
physical and conceptual-relational matching problem types, $F(1,3)=2.26, p=.23$. Hence, these animals were as competent at matching relations between relations as they were at matching on the basis of physical similarity. This equivalency of performance occurred despite the fact that the chimpanzees were not differentially rewarded during conceptual-relational matching trials, whereas they were during physical matching trials.

The ANOVA also permitted us to explore the possibility that performances on conceptual-relational matching trials resulted from associative rather than conceptual processes. Specifically, an improvement in performance across the two sessions for each problem might be expected if explicit associations between stimulus elements were learned. This would be reflected in either a between-session main effect, $F(1,3=9.00, p=-06$, or a Session $\times$ Problem Type (physical vs. conceptual) interaction, $F(1,3)=$ $1.12, p=.37$; neither of these were significant. Furthermore, if conceptual matching of relations was influenced by a learning set phenomenon, then correct performances should further increase not only across sessions but also between problems. This would be reflected as a significant main effect of problem set number; this was not the case, $F(2,6)=0.23, p=.80$.

We looked also at performances across eight-trial blocks within each session. If performances were mediated by stimulus associations, one might expect performance to improve across blocks. Although there was a significant main effect for trial blocks, $F(1,3)=14.49, p=.032$, it reflected instead a deterioration in performance from Block 1 to Block 2. Such a within-session performance decrement might be expected if initial matching judgments were based on relative familiarity versus novelty of stimulus pairs. There was, however, neither a Block X Session, $F(1,3)=0.02, p=.90$, nor a Block $\times$ Matching Problem Set, $F(2,6)=1.02, p=.42$, interaction. We conclude, therefore, that the decrement in performance between trial blocks was not caused by relative familiarity-novelty judgments. The fact that the block effect persisted across problems, regardless of matching problem type (conceptual vs. physical), suggests that the performance decrements resulted from a generalized deterioration in attention, reflecting increased fatigue or boredom with the task. This conclusion is supported by the finding that the decrease in performance over the two eight-trial blocks, within sessions, occurred independently of either physical or conceptual-relational matching judgments and, therefore, independently of whether correct responses were differentially or nondifferentially rewarded.

\section{Further Associative Analyses}

These analyses argue against an associative interpretation of the data from conceptual-relational matching trials. This conclusion was further reinforced by an analysis of conceptual matching errors in Problem Set 3.

Object-specific associative valence. As previously noted, the sample pairs in Problem Set 3 had also been used as samples in Problem Sets 1 and 2. Hence, Problem Sets 1 and 2 provided opportunities for the chimpanzees to develop associations between single objects in the sample and the correct alternative. For example, in Problem Set 1 , Object $A$ in the sample pair AA could have become positively associated with Object B of the correct BB alternative. If this had been the case, then performances in Problem Set 3 would have been adversely affected given our rules for constructing stimulus pairs.

In Problem Set 3, consider conceptual matching trials in which AA was the sample. Stimulus Item B, contrary to Problem Set 1, was now a member of the incorrect $\mathrm{BH}$ alternative (see Table 1 ). The other stimulus item, $\mathrm{H}$, was associatively neutral with respect to the sample AA objects because Item $\mathrm{H}$ and Object $A$ had never appeared previously together in the same problem. Similarly, the correct CC alternative consisted of Stimulus Item $C$, which was in the incorrect CD alternative associated with the AA 
sample in Problem Set 1 . Hence, Item $C$ might have become negatively associated with Object $A$ in Problem Set 1.

Table 2. Association Valence Values of Objects in Problem Set 3

\begin{tabular}{|c|c|c|c|c|c|c|c|c|}
\hline Trial & & & & & & Nor & tity & \\
\hline Sample pair & & & & & & & & \\
\hline Alternative pairs & (CC) & $\mathrm{BH}$ & (CC) & $\mathrm{BH}$ & $\mathrm{CC}$ & $(\mathrm{BH})$ & $\mathrm{CC}$ & $(\mathrm{BH})$ \\
\hline $\begin{array}{l}\text { Valence of objects in Problem } \\
\text { Set } 3 \text { Alternatives }\end{array}$ & & & & & & & & \\
\hline Object specific ${ }^{b}$ & 00 & $0+$ & -- -- & +0 & 00 & o -- & ++ & -0 \\
\hline Conditional associative $^{c}$ & -- -- & ++ & -- -- & ++ & ++ & -- -- & ++ & -- -- \\
\hline
\end{tabular}

Note. Pairs in parentheses indicate correct alternative choices. Individual letters within a pair refer to discrete objects (e.g., A = yellow talcum powder can and B = green tin cup.

\footnotetext{
aValence: Effect of prior associations (from Problem Sets 1 and 2) on probability of choice in current problem. "+" indicates instances in which past associations bias a chimpanzee toward choice of the object. "- indicates instances in which past associations bias a chimpanzee against choosing that object (see text for details), "o" indicates instances in which there is no valence associated with an object.

${ }^{b}$ Effect of prior associations with objects in current sample.

${ }^{\mathrm{c}}$ Effect of prior status as an object in the correct or incorrect alternative, given sample relation (identity or nonidentity) in current problem.
}

In Problem Set 3 then, there was a historical positive associative valence between Sample Object $A$ and Object $\mathrm{B}$ of the incorrect $\mathrm{BH}$ alternative. One might predict that this positive associative valence would bias an animal toward choosing the incorrect nonmatching pair. There was also a historical negative associative valence between Sample Object $A$ and both objects of the correct $C C$ alternative pair. This negative associative valence could bias an animal against choosing the correct stimulus pair. The respective positive $(+)$ and negative $(-)$ associative valences between all specific sample and alternative objects in Problem Set 3 are indicated in Table 2 under "object specific."

Conditional associative valences. Problem Set 3 allowed us also to probe for prior conditional associations between the relation instantiated by samples (i.e., identity or nonidentity) and specific items within the images of the alternative stimulus pairs. For example, in Problem Set 1 an animal may have chosen correctly not by matching relations with relations but by using an associative strategy such as "If identity, choose B" and "If nonidentity, choose C." Given our rules for constructing sample and alternative stimulus pairs in Problem Set 3, such a strategy would have adversely affected conceptual matching performances.

For example, in Problem Set 3, Objects B and H, which had previously been associated with AA and GG identity samples, respectively, were now combined to form the nonidentity alternatives (see Table 1). Likewise, Object $C$, which had been associated previously with the nonidentity EF sample, was now used in conjunction with identity samples in Problem Set 3.

In Problem Set 3 then, there were historical positive and negative conditional associative valences between sample relations of identity and nonidentity and individual items within the alternative stimulus pairs. These valences respectively could bias the animals toward choosing the incorrect and against choosing the correct alternatives. The respective conditional positive $(+)$ and negative $(-)$ associative valences between sample relations and alternative object images in Problem Set 3 are indicated in Table 2 under "conditional associative." 
The probability of conceptual matching errors was examined as a function of the total object-specific and conditional associative valences inherent within each alternative pair as shown in Table 2. Positive (+) valence biased animals toward choosing an incorrect nonmatching alternative, and negative (-) valence biased animals against choosing the correct matching alternative. Neutral valences (0) were assumed to be bias free.

The total conditional associative valence was equivalent in all trials of Problem Set 3 . There were two positive and two negative valences. As shown in Table 2, however, the total object-specific associative valence was either one or three. In the former case, there was either one positive or one negative valence value associated with the alternatives. In the latter case, the three valence values consisted of either two positive valences and one negative valence or, alternatively, two negative valences and one positive valence. Overall total valence values (five or seven) for each pair of stimulus alternatives were determined by summing both the object-specific and conditional associative valences within each pair of alternatives.

Interference in Problem Set 3 from a prior strategy of matching relations with specific objects in Problem Sets 1 and 2 might be expected, particularly in those animals previously trained on the conditional samedifferent discrimination task described previously. Results from a chi-square analysis of error frequencies, however, revealed no significant relationship between the specific or overall associative valence values and sample type $\left(x^{2}=2.35\right)$. Thus, this analysis revealed that the animals' performance on conceptualrelational matching trials in Problem Set 3 was not controlled by either object-specific or conditional associative factors. Regardless of valence, the total number of errors on conceptual-relational matching problems was low, and although the proportion of total errors that occurred on nonidentity trials was .57, this value did not differ significantly from $.50(z=.71)$. Hence, the animals' error choices were not systematically biased toward the choice of identity or nonidentity alternative pairs (cf. Oden et al., 1990, Exp. 3).

\section{Analysis of Trial 1 Performances}

The evidence presented thus far points to the control of performances on conceptual matching by judgments of relational equivalence. Correct performance on Trial 1 of a transfer problem is one of the more compelling measures of generalized matching ability (e.g., Oden et al., 1988, 1990). The correct Trial 1 performance across physical matching sessions for all problems was $83.3 \%$ overall $(p<.01$; binomial test). The correct Trial 1 performance across conceptual-relational matching sessions for all problems was, overall, $87.5 \%(p<.001$; binomial test). Trial 1 data for individual animals over all sessions within all three problems are shown in Table 3.

Physical matching sessions. Darrell and Sheba were correct on Trial 1 of each of the six physical matching sessions in Problem Sets 1, 2, and 3. Kermit made a single physical matching error on Trial 1 of the second session of Problem Set 2. Sarah committed three physical matching errors on Trial 1 . She made a Trial 1 error on Session 1 of Problem Set 1, Session 2 of Problem Set 2, and Session 1 of Problem Set 3.

Conceptual matching sessions. Only Sarah was errorless on Trial 1 of each of the six conceptualrelational matching sessions in Problem Sets 1, 2, and 3. Darrell, Sheba, and Kermit each made a single Trial 1 error in the three problem sets. Darrell was incorrect on Trial 1 of the first session of Problem Set 2. Sheba made an incorrect conceptual matching choice on Trial 1 of Session 2 in Problem Set 3. Kermit made a Trial 1 conceptual matching error on Session 2 of Problem Set 1. 
Table 3. Trial 1 Responses in Problem Sets 1 to 3

\begin{tabular}{|c|c|c|c|c|c|c|c|c|c|c|}
\hline \multirow[b]{2}{*}{ Problem set and session } & \multicolumn{5}{|c|}{ Conceptual problems } & \multicolumn{5}{|c|}{ Physical problems } \\
\hline & Darrell & Sheba & Sarah & Kermit & $\begin{array}{c}\text { All } \\
\text { animals }\end{array}$ & Darrell & Sheba & Sarah & Kermit & $\begin{array}{c}\text { All } \\
\text { animals }\end{array}$ \\
\hline \multicolumn{11}{|l|}{ Problem Set 1} \\
\hline Session 1 & 1 & 1 & 1 & 1 & 4 & 1 & 1 & 0 & 1 & 3 \\
\hline Session 2 & 1 & 1 & 1 & 0 & 3 & 1 & 1 & 1 & 1 & 4 \\
\hline \multicolumn{11}{|l|}{ Problem Set 2} \\
\hline Session 1 & 0 & 1 & 1 & 1 & 3 & 1 & 1 & 1 & 1 & 4 \\
\hline Session 2 & 1 & 1 & 1 & 1 & 4 & 1 & 1 & 0 & 0 & 2 \\
\hline \multicolumn{11}{|l|}{ Problem Set 3} \\
\hline Session 1 & 1 & 1 & 1 & 1 & 4 & 1 & 1 & 0 & 1 & 3 \\
\hline Session 2 & 1 & 0 & 1 & 1 & 3 & 1 & 1 & 1 & 1 & 4 \\
\hline All sessions & 5 & 5 & 6 & 5 & $21^{\star *}$ & 6 & 6 & 3 & 5 & $20^{*}$ \\
\hline
\end{tabular}

Note. 1 = correct choice; $0=$ error.

${ }^{\star} p<.01$, binomial test. ${ }^{\star *} p<.001$, binomial test.

The mean overall correct performance level for the three sessions that Darrell, Sheba, and Kermit began with an incorrect conceptual-relational matching choice was $81.25 \%(p<.001$, binomial test). In the corresponding sessions in which they were correct on Trial 1, Darrell and Sheba's mean correct conceptual-relational matching performances were both $82.5 \%(p<.001$, binomial test). Kermit's corresponding performance level was $85 \%(p<.001$, binomial test). These Trial 1 performance data from each session of all problem sets revealed that rewarding an incorrect choice on Trial 1 of a conceptualrelational matching problem had no measurable influence on subsequent performance. As noted, overall conceptual matching accuracy on Trial 1 for all problem sets was well above chance and, hence, further evidence that these chimpanzees approached the problems with a conceptual response strategy of matching relations between relations that generalized across matching exemplars.

\section{Discussion}

\section{Conceptual Matching Without Language Training}

Four of 5 chimpanzees conceptually matched relations between relations in three problem sets at well above chance levels in the absence of any explicit training via differential feedback on each trial. Furthermore, they performed correctly at levels that were statistically indistinguishable from their physical matching performances in the problem sets under conditions of differential reward.

Three of the animals spontaneously matched relations between relations despite their never having received any training in the language system experienced years ago by Sarah, a fourth chimpanzee who also had a history of conceptual problem solving. The fifth chimpanzee, Bobby, a juvenile male, failed to achieve criterion not only on conceptual but also on physical matching trials. This animal was naive with respect to language training, numeric problem solving, and the conditional discrimination task experienced by the other animals. Bobby also had not had explicit training on object-image recognition.

The remainder of this discussion addresses several issues. First, we discuss the question of whether the conceptual-relational matching problems were solved using associative processes. Second, we consider two hypotheses presented as challenges to D. Premack's original assertions as to the necessary 
importance of language training for acquisition of a broadly construed conceptual matching ability. One hypothesis is that conceptual-relational matching can emerge from nothing more than a rich and varied history of training and testing (Roitblat, 1985). A second hypothesis is that conceptual matching of relations between relations is simply a procedural skill resulting from extensive experience with differential reinforcement. We argue that neither of these hypotheses can account for the results of the present study. Finally, we discuss what we consider to be the necessary conditions responsible for the spontaneous expression of conceptually driven abstract relational equivalence judgments.

\section{Inadequacies of Associative Interpretations}

There is good evidence that many species can solve problems involving equivalence judgments using strategies other than a conceptual judgment that an item or relation (e.g., identity or nonidentity) is the same as another (D. Premack, 1983a; Thompson, 1995). One such process might be stimulus-specific associations between individual objects making up the samples and alternatives. That is, an animal's performance may be based either wholly or in part on the simple associative rule "If A then B" and "If $E$ then C." For example, the procedure used by Smith et al. (1975) of differentially rewarding correct matches and not rewarding mismatches left open the possibility of their chimpanzees learning their tasks in this manner.

An animal's dependence on such stimulus-specific attributes in a matching task may be revealed when it fails to transfer its performance to novel items (e.g., Oden et al., 1990). Typically, in cases in which differential reward is used, correct performance on Trial 1 with any given stimulus set is a primary criterion for inferring a conceptual basis to performance. No such data were reported by Smith et al. (1975), whose only reported control was for position effects of a correct alternative for a given sample.

Another possible process is a conditional response rule, as discussed previously, in which the choice of a particular alternative was determined by the relationship instantiated by the sample. That is, an animal's performance in Problem Set 1 may have been based in part on the rule "If identity sample, then choose Item B" and "If nonidentity sample, then choose Item C."

In the case of our animals that matched relations between relations, there was no evidence of associative influences on performances in the three problem sets. This conclusion is based on three lines of evidence. First, performance levels did not improve across either of the two sessions within each problem set or across the three problem sets. Although there was a significant performance decrement within each 16-trial session, it was attributable to motivational or attentional factors rather than a reflection of associative processes. This conclusion was based on the fact that the decrement was observed during both physical and conceptual relational matching sessions. Hence, the decrement occurred independently of whether or not responses were differentially reinforced. Furthermore, the magnitude of the decrements was consistent throughout the study.

The second line of evidence against interpreting the chimpanzees' performances within an associative framework is found in the results from Problem Set 3. Alternative pairs in Problem Set 3 were constructed such that the associative histories of each within-pair element would lead to performance decrements if past performances were based on either simple or complex associative rather than conceptual judgments. No such decrements were observed, indicating that the animals were matching relations between relations conceptually.

Finally, the third line of evidence against an associative interpretation of the conceptual-relational matching results is provided by an examination of matching performances on Trial 1 of each session within each problem. The results revealed that correct Trial 1 matches were significantly above chance 
and, furthermore, correct performances on subsequent trials did not vary systematically with whether or not a correct or incorrect choice was made on Trial 1.

Overall, then, there is nothing to suggest that conceptual-relational matching by these 4 animals was mediated by associative processes. When these chimpanzees matched relations, they were, in essence, indicating that they judged "the relation exemplified by this (alternative) image-pair is the same as that exemplified by the sample object-pair." What we do not know is the specific relational dimension within pairs (e.g., AA and CD) that they were using to denote sameness between pairs. It could have been, for example, the relational dimension denoted by the experimenters (i.e., one of reflexive identity [A is A] vs. nonidentity [C is not $D$ ]). Alternatively, the within-pair stimulus dimension might have been one of symmetry (A maps onto A) versus asymmetry (C does not map onto D). Regardless of the functional within-pair relational dimension, the resulting matching judgment of relational equivalence between relations $(A A=B B ; C D=E F)$ could not be based on physical dimensions of color, shape, or size.

\section{Is Language Training a Necessary Condition for Conceptual Matching?}

What are the necessary and sufficient conditions for conceptual matching of relations between relations? D. Premack's early discussions $(1983 a, 1983 b, 1984)$ emphasized the facilitative effect of language training per se. In this section, we discuss two alternative hypotheses, namely test sophistication and persistent or dogged training with differential reinforcement.

Test sophistication hypothesis. Some critics of D. Premack's early position (e.g., Roitblat, 1985) proposed that an overall rich and varied history of problem solving in general, rather than language training per se, enabled Premack's chimpanzees to solve conceptual matching and related analogical problems. The argument here is that exposing animals to a variety of different problem types creates a test-sophisticated animal better prepared to engage novel situations and problems with a range of hypotheses at various levels of stimulus complexity (i.e., from concrete physical to conceptual and abstract).

A similar argument could be made concerning the successful conceptual matching of relations between relations by the 3 language-naive animals in the present study. That is, prior training on the conditional discrimination task involving concrete tokens and relations may not have been critical. Rather, this training may simply have been one experience among many that collectively, and through some unspecified process, enhanced conceptual relational competence. The appeal of the test sophistication argument is weakened, however, by D. Premack's reports $(1976,1983 a, 1983 b)$ of test-sophisticated but language-naive animals that failed to match relations between relations and to perform related analogical problem-solving tasks.

To our knowledge, the definitive empirical test for the causal effect of token training per se, independently of language training, has not been conducted. Such a test would entail two groups of experimentally naive chimps, one of which is made test sophisticated via exposure to a rich variety of problem-solving tasks-including those involving relations and analogical reasoning-and another group given token training alone. Subsequently, both groups would be tested under conditions of nondifferential reinforcement on a conceptual-relational matching task. If, as suggested by Thompson and Oden (1993), token training alone is necessary for the emergence of conceptual problem solving, then the latter group of chimpanzees, but not the former, should pass the test.

Dogged-training hypothesis. As noted early in this article, D. Premack (1988) briefly mentioned one study that suggested that conceptual matching can be achieved by training of a "nonlinguistic" character. In this report, he referred to the effects of persistent dogged training on a problem in which chimpanzees were required to match like proportions of different objects (e.g., to match $1 / 2$ apple to $1 / 2$ container of liquid). 
Those results, however, differ fundamentally from those of the present study and from the previously reported effects of language training. The performance of the animals hi the proportional matching task did not transfer to novel problems involving other forms of relational judgments like, presumably, conceptual-relational matching to sample (D. Premack, 1988). This was not the case for Premack's language-trained animals that did generalize their relational problem-solving skills across several different nonlinguistic domains, including conceptual-relational matching (cf. D. Premack, 1983a, 1983b, 1984).

Likewise, the chimpanzees in the present study spontaneously solved a novel problem involving relational judgments. Nowhere in their training history had they been explicitly trained or tested on a conceptual task involving the matching of relations between relations. Their prior experience, however, did entail the matching of identity and nonidentity stimulus pairs with concrete tokens. The functional role of concrete tokens as symbols for relational information was likely further reinforced by their additional experience matching Arabic numerals to arrays of objects (Boysen, 1993; Boysen \& Bernston, 1989).

\section{Necessary and Sufficient Prior Conditions}

We conclude that the test sophistication and dogged training hypotheses are insufficient to account for the evidence of spontaneous conceptual-relational matching demonstrated in this study and elsewhere. We turn now to consider other alternatives as to what might be the necessary and sufficient prior conditions.

Experience with discursive strings of symbols. One interpretation championed by D. Premack in his earlier writings focuses on experience judging the equivalence between a string of potentially linguistic elements and the propositional conditions that they represent (D. Premack, 1983a, 1983b, 1984,1986). The second approach is to focus on the functional role of concrete tags or labels for same-different relations (Thompson \& Oden, 1993). Despite his more recent emphasis on the functional role of "words" or tokens per se, D. Premack continues to entertain the possibility that experience with other aspects of language training (e.g., lexicon and sentences) "may be an indirect prerequisite" (Premack, 1988, p. 53) for analogical reasoning about relations between relations.

The present data indicate that experience with linguistic discursive strings of tokens is not necessary for problem solving involving judgments about the equivalence of relations in a matching task. As previously noted, 3 of the chimpanzees that matched relations between relations in the present study had not experienced explicit training involving the use of discursive strings of tokens. They were experienced, as noted, with labeling numeric displays of objects with Arabic numerals (e.g., 3 is $X X X$ and $X X X$ is 3 ) and with labeling pairs of objects as being either the same or different (i.e., "If identity, choose heart" and "If nonidentity, choose diagonal"). The remaining critical issue, then, is whether this prior experience with the labeling of referents and relations with tokens (cf. Danto, 1983, D. Premack, 1983b) was the critical causal factor underlying the spontaneous emergence of conceptual-relational matching capabilities.

Experience with token training. Both D. Premack (1988) and Thompson and Oden (1993) suggested that the facultative effects of token training or labeling might result from a broadly construed attentional shift in the processing hierarchy an animal brings to bear in problem-solving situations. The natural predisposition to attend to physical sensory features on which computational processes are applied presumably is displaced by a tendency to process abstract relational aspects of stimulus arrays that are perceived directly (Oden et al., 1990).

We suspect that such an attentional shift was achieved in D. Premack's experiment (1988) involving the persistent dogged training of chimpanzees on a proportions matching task. As noted previously, however, the effect of this training was specific to the proportions problem. In contrast, the token-training 
experiences of the chimpanzees in the present study were much more broadly effective. Apparently, they enabled the chimpanzees to subsequently match relations between relations spontaneously in the absence of any explicit token or symbol.

Hence, although persistent training over thousands of differentially reinforced trials may facilitate an attentional shift, the effect is not broadly construed. It constrains success to the specific type of problem encountered in the original training. Prior experience with arbitrary tokens that are potentially labels for referent items or relations, however, is not similarly constrained; its effect is broadly construed.

Apparently, then, some kinds of nonlinguistic experiences "upgrade a mind" (D. Premack \& Premack, 1983) in ways that other kinds do not. The performance of the present animals is unequivocally matched only by those animals in D. Premack's laboratories that had enjoyed the benefits of language training. The common experiential denominator shared by his language-sophisticated animals and the languagenaive animals in the present study is experience with tokens. However, why should training with tokens such as symbols for "same and different" or numeric displays cause an animal not only to shift its attention to prepositional (relational) levels of processing but also to generalize this attentional shift to novel situations and problem types in a way not observed with other procedures? (See also Herman, Hovancik, Gory, \& Bradshaw, 1989; Savage-Rumbaugh, 1986; Savage-Rumbaugh et al., 1993.)

\section{Functional Role of Tokens}

Thompson and Oden (1993) proposed that any training history involving the functional correspondence between an arbitrary cue or token and an abstract relation is sufficient to enable a chimpanzee to match relations between relations. They based their hypothesis on the suggestion that a critical function of the token is to provide an animal with a concrete icon for encoding an otherwise abstract prepositional representation. That is, the token or tag has the retrieval function of a word (cf. D. Premack, 1990, p. 209; D. Premack \& Premack, 1994, p. 98). An English-speaking human seeing or hearing the word rabbit retrieves the properties of that animal that can be represented iconically or discursively by other words. However, the functional relationship between words and the information they retrieve is reciprocal or reflexive. Hence, if one now hears or sees the referent, one can retrieve the word, or even a discursive string of words, describing that object, event, or relationship (e.g., "See 'XXX', 'think three'"). That is, the token or tag "objectifies" a relationship (Kluver, 1933/1961) per se independently of any particular exemplar. In this sense, then, tokens are analogous to words. They serve as concrete symbols or icons that anchor propositional knowledge.

Conceptual-relational matching as covert symbol matching. According to the taxonomic model of matching proposed by Thompson and Oden (1996), these symbolic tokens provide the necessary iconic representational basis on which the multiple encoding and computational steps mandated by the conceptual-relational matching procedure can occur. Hence, in the present study, one can hypothesize the following sequence of computational and representation events.

First, the chimpanzee detects the within-pair relation instantiated, for example, by an AA (identity) or a CD (nonidentity) sample. This evokes an internal iconic representation of the concrete token (e.g., "heart" or "diagonal") that symbolically codes identity and nonidentity, as learned previously in an unrelated task. Likewise, these icons symbolizing the relations identity and nonidentity, respectively, will be evoked when the chimpanzee next detects the within-pair relations instantiated by the BB and EF alternatives presented on the computer monitor.

The chimpanzee can now covertly match these representational icons (e.g., heart and diagonal) against the symbolic representation of heart or diagonal evoked by the sample. Thompson and Oden (1996) 
hypothesized that it is only at this point in the sequence of computational and encoding processes that an animal is in the position to judge one relation as being the same as another relation (cf. Table 1 in Thompson \& Oden, 1996).

Conceptual-relational matching is made possible by the chimpanzee's representational capacity to reencode abstract relations, which are instantiated by physically disparate sample and alternative pairs, into iconically identical symbols. It follows from this analysis that prior token training reduces conceptualrelational matching to a task that is functionally equivalent to physical matching. Both types of equivalence judgment (i.e., one object to another and one relation to another) become the product of covert concrete icon matching.

Conclusion. In short, we believe experience with symbolic tokens per se produces a system for universal computation in the chimpanzee, as it does also for the human child, if not other species. The present results, taken in the context of previous studies with language-naive chimpanzees (e.g., Oden et al., 1990), also support the theoretical assumption that symbols are "in the world" first and only later "in the head" (Hutchins, 1995). Furthermore, the chimpanzee's "upgraded mind," like that of a child, is the product of a historical process involving the external organization of symbolic experiences by other beings and, in the chimpanzee's case, another species (D. Premack, 1988, 1990; Rumbaugh \& SavageRumbaugh, 1992).

\section{References}

Boysen, S.T. (1992). Pongid pedagogy: The contribution of human-chimpanzee interactions to the study of ape cognition. In H. Davis \& D. Balfour (Eds.), The inevitable bond: Examining scientist-animal interactions (pp. 205-217). New York: Cambridge University Press.

Boysen, S. T. (1993). Counting in chimpanzees: Nonhuman principles and emergent properties of number. In S. T. Boysen \& E. J. Capaldi (Eds.), The development of numerical competency: Animal and human models (pp. 39-59). Hillsdale, NJ: Erlbaum.

Boysen, S. T., \& Bernston, G. G. (1989). Numerical competence in a chimpanzee (Pan troglodytes). Journal of Comparative Psychology, 103, 23-31.

Danto, A. C. (1983). Images, labels, concepts, and propositions: Some reservations regarding Premack's "abstract code." The Behavioral and Brain Sciences, 6, 143-144.

Herman, L. M., Hovancik, J. R., Gory J. D., \& Bradshaw, G. L. (1989). Generalization of visual matching by a bottlenosed dolphin (Tursiops truncatus): Evidence for invariance of cognitive performance with visual and auditory materials. Journal of Comparative Psychology, 15, 124-136.

Hutchins, E. (1995). Cognition in the wild. Cambridge, MA: MIT Press.

Kluver, H. (1961). Behavior mechanisms in monkeys. Chicago: University of Chicago Press. (Original work published 1933)

Nissen, H. W., Blum, J. S., \& Blum, R. A. (1948). Analysis of matching behavior in chimpanzees. Journal of Comparative and Physiological Psychology, 41, 62-74.

Oden, D. L., \& Thompson, R. K. R. (1992). The role of social bonds in motivating chimpanzee cognition. In H. Davis \& D. Balfour (Eds.), The inevitable bond: Examining scientist-animal interactions (pp. 218-231). Cambridge, England: Cambridge University Press.

Oden, D. L., Thompson, R. K. R., \& Premack, D. (1988). Spontaneous transfer of matching by infant chimpanzees (Pan troglodytes). Journal of Experimental Psychology: Animal Behavior Processes, 14, 140-145.

Oden, D. L., Thompson, R. K. R., \& Premack, D. (1990). Infant chimpanzees (Pan troglodytes) spontaneously perceive both concrete and abstract same/different relations. Child Development, 61, 621-631.

Premack, A. J., \& Premack, D. (1972). Teaching language to an ape. Scientific American, 227, 92-99. 
Premack, D. (1976). Intelligence in ape and num. Hillsdale, NJ: Erlbaum.

Premack, D. (1983a). Animal cognition. Annual Review of Psychology, 34, 351-362.

Premack, D. (1983b). The codes of man and beast. The Behavioral and Brain Sciences, 6, 125-137.

Premack, D. (1984). Upgrading a mind. In T. G. Bever, J. M. Carroll, \& L. A. Miller (Eds.), Talking minds: The study of language in cognitive science (pp. 181-206). Cambridge, MA: MIT Press.

Premack, D. (1986). Gavagai! Or the future history of the animal language controversy. Cambridge, MA: Bradford, MIT Press.

Premack, D. (1988). Minds with and without language. In L. Weiskrantz (Ed.), Thought without language (pp. 46-65). New York: Oxford University Press.

Premack, D. (1990). Words: What are they, and do animals have them? Cognition, 37, 197-212.

Premack, D. (1992). On the origins of domain-specific primitives. In H. L. Pick, Jr., P. van den Broek, \& D. C. Knill (Eds.), Cognition: Conceptual and methodological issues (pp. 189-212). Washington, DC: American Psychological Association.

Premack, D., \& Premack, A. J. (1983). The mind of an ape. New York: Norton.

Premack, D., \& Premack, A. J. (1994). How "theory of mind" constrains language and communication. In D. C. Gajdusek, G. M. McKehann, \& L. C. Bolis (Eds.), Discussions in neuroscience, Vol. X: Evolution and neurology of language (pp. 93-105). New York: Elsevier Science.

Roitblat, H. L. (1985). Introduction to comparative cognition. New York: Freeman.

Rumbaugh, D., \& Savage-Rumbaugh, E. S. (1992). Cognitive competencies: Products of genes, experience, and technology. In T. Nishida, W. C. McGrew, P. Marler, M. Pickford, \& F. B. M. de Waal (Eds.), Topics in primatology, Vol. 1: Human origins (pp. 293-304). Tokyo: University of Tokyo Press.

Savage-Rumbaugh, S. (1986). Ape language: From conditioned response to symbol. New York: Columbia University Press.

Savage-Rumbaugh, S., Murphy, J., Sevcik, R. A., Brakke, K. E., Williams, S. L., \& Rumbaugh, D. M. (1993). Language comprehension in ape and child. Monographs of the Society for Research in Child Development, 58(3-4, Serial No. 233).

Smith, H. J., King, J. E., Witt, E. D., \& Rickel, J. E. (1975). Sameness-difference matching from sample by chimpanzees. Bulletin of the Psychonomic Society, 6, 469-471.

Thompson, R. K. R. (1995). Natural and relational concepts in animals. In H. Roitblat \& J. A. Meyer (Eds.), Comparative approaches to cognitive science (pp. 175-224). Cambridge, MA: Bradford/MIT Press.

Thompson, R. K. R., \& Oden, D. L. (1993). "Language training" and its role in the expression of tacit propositional knowledge in chimpanzees (Pan troglodytes). In H. L. Roitblat, L. M. Herman, \& P. E. Nachtigall (Eds.), Language and communication: Comparative perspectives (pp. 365-384). Hillsdale, NJ: Erlbaum.

Thompson, R. K. R., \& Oden, D. L. (1996). A profound disparity revisited: Perception and judgment of abstract identity relations by chimpanzees, human infants, and monkeys. Behavioral Processes, 35, 149-161. 